\title{
Feasibility of emergency department point-of-care ultrasound for rib fracture diagnosis in minor thoracic injury
}

\author{
Élizabeth Lalande, $\mathrm{MD}^{*}$; Chantal Guimont, $\mathrm{MD}, \mathrm{PhD}^{*^{\dagger}}$; Marcel Émond, $\mathrm{MD}, \mathrm{MSc}^{* *}$; \\ Marc Charles Parent, MD*; Claude Topping, MD*; Brice Lionel Batomen Kuimi, $\mathrm{MSc}^{\ddagger}$; \\ Valérie Boucher, $\mathrm{BA}^{\ddagger}$; Natalie Le Sage, MD, $\mathrm{PhD}^{*^{\ddagger}}$
}

\section{ABSTRACT}

Objectives: The main objective of this study was to evaluate the feasibility of emergency department (ED) point-of-care ultrasound (PoCUS) for rib fracture diagnosis in patients with minor thoracic injury $(\mathrm{mTI})$. Secondary objectives were to 1) evaluate patients' pain during the PoCUS procedure, 2) identify the limitations of the use of PoCUS technique, and 3) compare the diagnosis obtained with PoCUS to radiography results.

Methods: Adult patients who presented with clinical suspicion of rib fractures after $\mathrm{mTI}$ were included. All patients underwent PoCUS performed by emergency physicians (EPs) prior to a rib view X-ray. A visual analogue scale (VAS) ranging from 0 to 100 was used to ascertain feasibility, patients' pain and clinicians' degree of certitude. Feasibility was defined as a score of more than 50 on the VAS. We documented the radiologists' interpretation of rib view X-ray. Radiologists were blinded to the PoCUS results.

Results: Ninety-six patients were included. A majority (65\%) of EPs concluded that the PoCUS technique to diagnose rib fracture was feasible (VAS score $>50$ ). Median score for feasibility was 63 . Median score was 31 (Interquartile range [IOR] 5-57) for patients' pain related to the PoCUS. The main limiting factor of the PoCUS technique was pain during patient examination (15\%).

Conclusion: PoCUS examination appears to be a feasible technique for a rib fracture diagnosis in the ED.

\section{RÉSUMÉ}

Objectifs: L'objectif principal de cette étude consistait à évaluer la faisabilité de l'échographie ciblée au département d'urgence (ÉDU) pour le diagnostic des fractures de côtes chez les patients ayant subi un traumatisme thoracique mineur (TTm). Les objectifs secondaires étaient les suivants : 1) évaluer la douleur au cours de la procédure de I'ÉDU, 2) identifier les limites de la technique de l'ÉDU dans ce contexte et 3) comparer le diagnostic obtenu avec I'ÉDU aux résultats de la radiographie.

Méthodes: Des patients adultes avec suspicion clinique de fractures de côtes suite à un TTm ont été recrutés prospectivement et l'ÉDU a été effectuée par les médecins d'urgence avant la radiographie. Une échelle visuelle analogue (EVA) graduée de 0 à 100 a été utilisée afin d'évaluer la faisabilité de la technique, la douleur des patients et le degré de certitude des cliniciens. La faisabilité a été définie comme un score de plus de 50 sur l'EVA. Les radiographies ont été interprétées par des radiologistes à l'aveugle des résultats de l'ÉDU.

Résultats: Quatre-vingt-seize patients ont été inclus. Une majorité $(65 \%)$ des médecins d'urgence a conclu que la technique de l'ÉDU pour diagnostiquer les fractures de côte était faisable (score EVA>50). Le score médian de faisabilité était de 63. Le score médian de la douleur des patients était de 31 (intervalle interquartile (IOR) 5-57). Le principal facteur limitant décrit par les médecins était la douleur lors de l'examen (15\%).

Conclusions: L'échographie ciblée au département d'urgence semble être une technique applicable pour le diagnostic de fractures de côtes.

Keywords: Rib fracture, point-of-care ultrasound, minor thoracic injury

\section{INTRODUCTION}

Rib fractures represent a frequent condition associated with minor thoracic injury (mTI). ${ }^{1}$ Previous studies have shown that more than a third of patients with minor blunt chest trauma may be suffering from rib

From the *Département de Médecine Familiale et Médecine d'Urgence, Université Laval, Quebec, QC; †Centre de recherche du CHU de Québec, Axe Reproduction, Santé et la Mère et de I’Enfant, Quebec, QC; and ¥Centre de recherche du CHU de Québec, Axe Santé des Populations et Pratiques Optimales en santé, Unité de recherche en Traumatologie - Urgence - Soins Intensifs, Quebec, QC.

Correspondence to: Natalie Le Sage, Université Laval, Centre de recherche du CHU de Québec, 1401, 18 rue, Québec, QC G1J 1Z4; Email: natalie. lesage@fmed.ulaval.ca 
fractures. ${ }^{1,2}$ These are associated with short- and longterm limitations such as acute pain and significant work absenteeism. ${ }^{3,4}$ Serious acute complications, such as pneumothorax, hemothorax, lung contusion, and flail chest, may result from rib fractures. ${ }^{2}$ Moreover, delayed complications, such as pneumothorax, hemothorax and pneumonia, may also following such injuries. ${ }^{5-7}$ These complications increase with the number of diagnosed rib fractures, ${ }^{2,6,7}$ as does mortality. ${ }^{6-8}$ In order to identify patients at risk of complications, an early and accurate diagnosis of rib fracture may be helpful.

In patients with a blunt chest trauma, studies have shown that clinical symptoms alone do not correlate well with the presence of rib fractures. ${ }^{9,10}$ Chest X-ray and rib views have a limited sensitivity to identify rib fractures and vary significantly among studies, reaching at most $50 \% .^{11,12}$ Computed tomography (CT) is sometimes considered as the gold standard to diagnose rib fractures, but it exposes patients to a significant amount of radiation. Furthermore, it is an imperfect imaging tool, because the axis of tomography images can miss fractures due to the particular rib cage anatomy. ${ }^{13}$

Since the last decade, point-of-care ultrasound (PoCUS) has become an important part of emergency physicians' (EP) daily practice, and its applications have become numerous. Studies have reported that ultrasound is more sensitive than $\mathrm{X}$-ray to detect rib fractures. ${ }^{11,14,15}$ As such, there may be potential benefits over plain radiography for performing rib PoCUS in mTI. Knowing the limits of existing imaging techniques used for the diagnosis of rib fractures and the growing availability of PoCUS in emergency departments (EDs), we conducted a prospective cohort study in which we hypothesized that rib PoCUS would be a feasible technique to diagnose rib fractures in patients with mTI. Secondary objectives were to 1) evaluate patients' pain during the PoCUS procedure, 2) identify the limitations of the PoCUS technique, and 3) compare the diagnosis obtained with PoCUS to radiography results.

\section{METHODS}

\section{Population}

This study took place in a tertiary trauma centre in the province of Quebec, Canada. Clinical suspicion of rib facture was the main inclusion criterion in patients age 18 years or older who sustained blunt thoracic trauma. A convenience sample was used. The exclusion criteria were defined as follows: delay in seeking care (more than 96 hours post-trauma), hemodynamic instability (defined as a heart rate of more than 100 beats per minute, saturation of less than $95 \%$, systolic pressure of less than $90 \mathrm{~mm} \mathrm{Hg}$, and respiratory rate of more than 20 per minute), a score of 14 or less on the Glasgow Coma Scale (GCS), patients suffering from significant traumatic injuries requiring treatment and hospitalization (mediastinal injury, spinal fractures, flail chest, significant hemothorax, and/or pneumothorax requiring tube thoracostomy).

\section{Ultrasound training}

Participating clinicians attended a 2-hour training session before the recruitment of patients began (see Supplementary Material). Two experienced PoCUS instructors taught participating EPs. The first hour allowed them to become familiarized with the study protocol, and the ultrasound technique to diagnose rib fractures was explained. During the second hour of this session, EPs were allowed to practice the ultrasound technique.

EPs were told to locate the point of maximal tenderness with palpation of the patient thorax. Then, the operator applied a high frequency linear ultrasound probe perpendicular to the long axis of the rib. A distinct shadowing posterior to the rib helped in differentiating the pleural line from the rib cortex. After adequately locating the rib, the probe was turned ninety degrees to allow the clinician to follow the rib cortex longitudinally; this appeared as a white, hyperechoic line. The clinician was asked to start screening $10 \mathrm{~cm}$ before the point of maximal tenderness and to finish $10 \mathrm{~cm}$ beyond, to ensure that no fracture would be missed. ${ }^{12}$ EPs were asked to stabilize the probe between the index and major fingers of the non-dominant hand. This made it possible to feel the intercostal spaces with the same index and major fingers, allowing the clinician to precisely screen the rib (Figure 1). The upper and lower adjacent ribs were also screened. A rib fracture was diagnosed when a discontinuity of the cortical alignment was observed, visualized as a gap through the hyperechoic cortical line of the rib.

\section{Data collection}

Sociodemographic and clinical characteristics were collected using a standardized form during the initial ED visit. Presence or absence of rib fractures was documented. 


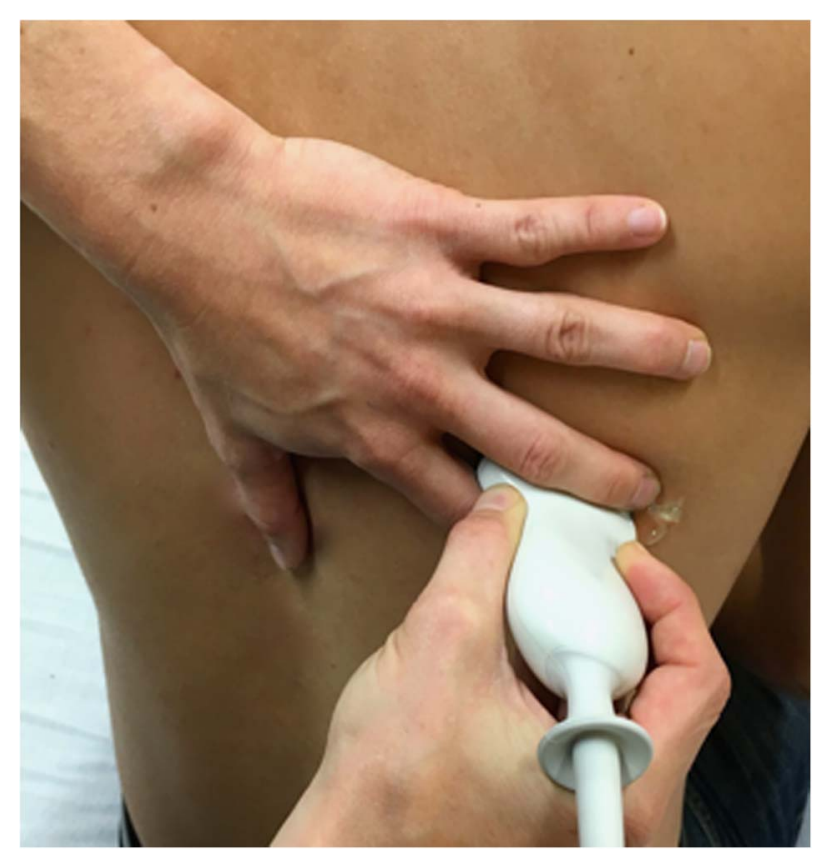

Figure 1. Rib point-of-care ultrasound technique.

EPs were blinded to the chest X-ray findings when they performed the rib PoCUS and when they filled out the study survey materials. Subsequently, a radiologist, blinded to the PoCUS results, interpreted the $\mathrm{X}$-rays, and results were compiled.

\section{Outcome measures}

The feasibility of PoCUS for the detection of rib fractures was mainly ascertained in terms of acceptability. ${ }^{16}$ The primary outcome was the feasibility score (FS), which was defined as a score of more than 50 measured on a visual analogue scale (VAS) completed by the EP who performed the PoCUS. This score allowed to evaluate the extent to which the technique could be performed in the context of the ED. The clinician was asked to rate the feasibility of the rib PoCUS technique, from 0 (extremely difficult) to 100 (extremely easy). There are no previous publications in the medical literature to provide a formal accepted definition of a feasibility score for rib PoCUS for fractures. A steering committee, which included five EPs experienced with PoCUS, was consequently formed in order to come to a consensus agreement for the main outcome. The threshold FS was defined by the steering committee as a score of more than 50 measured on a VAS completed by the EP who performed the PoCUS.
Furthermore, to assess acceptability, patients were asked to rate their degree of pain associated with the technique using a VAS ranging from 0 (no pain) to 100 (unbearable pain). Technical limitations were classified as present or absent. Anticipated limitations, such as obesity, technical difficulty, and patient's pain during the technique, were available answers but clinicians could also specify any other limitations regarding the PoCUS that they performed. Further comparisons were made using a database in which formal chest X-ray interpretation and PoCUS results were compiled for each patient. Patient charts were also reviewed in order to complete the database with this information when available.

\section{Statistical analysis}

All data were described with measures of proportion, median, and dispersion with interquartile range. All analyses were conducted using the Statistical Analysis System software, version 9.4 (SAS Institute Cary, NC, USA). Cohen tags ${ }^{17}$ and Pearson correlation coefficient were used for statistical analysis purposes.

\section{Ethics}

The Research Ethics Boards of the Centre Hospitalier Universitaire (CHU) de Québec - Université Laval approved the study protocol, and written consent was obtained for all recruited patients.

\section{RESULTS}

Ninety-six patients were recruited from November 2011 to December 2013, and 11 out of 24 (46\%) full-time EPs participated in this study. All recruiting clinicians had a mean experience of 9 years with bedside ultrasound, although none of the participating EPs had experience with rib PoCUS.

The majority of patients were men $(69.8 \%)$. The main mechanisms for trauma were fall from height $(60 \%)$, sport $(12 \%)$, motor vehicle collision $(8 \%)$, and direct blow (7\%). Median time between trauma and ED consultation was 18.9 hours (IQR 5.6-47.3). These results and others, including vital signs on arrival, are further described in Table 1.

A majority (65\%) of EPs concluded that the PoCUS technique to diagnose rib fracture was feasible (FS > 50) with a median score of 63 (Table 2). Patients' pain related to the PoCUS examination was relatively 


\begin{tabular}{|c|c|}
\hline \multicolumn{2}{|l|}{ Baseline characteristics of participants } \\
\hline Men (n [\%]) & $67(69.8)$ \\
\hline Age (years) (median and IQR) & $54(38-69)$ \\
\hline \multicolumn{2}{|l|}{ Trauma mechanism (n [\%]) } \\
\hline Fall from height & $58(60.4)$ \\
\hline Sport accident & $11(11.5)$ \\
\hline Motor vehicle accident & $11(11.4)$ \\
\hline Direct blow & $7(7.3)$ \\
\hline Fall from more than height & $5(5.2)$ \\
\hline Cough & $2(2.1)$ \\
\hline Others & $2(2.1)$ \\
\hline $\begin{array}{l}\text { Delay since traumatism (hours) } \\
\text { (median and IQR) }\end{array}$ & $18.9(5.6-47.3)$ \\
\hline \multicolumn{2}{|l|}{ Vital signs (median and IQR) } \\
\hline Heart rate (rate/min) & 79 (70-89) \\
\hline Respiratory rate (rate/min) & $16(16-20)$ \\
\hline Saturation (\%) & 97 (96-99) \\
\hline Systolic arterial pressure $(\mathrm{mm} \mathrm{Hg})$ & 147 (128-159) \\
\hline Diastolic arterial pressure $(\mathrm{mm} \mathrm{Hg})$ & $80(72-90)$ \\
\hline
\end{tabular}

\begin{tabular}{|c|c|}
\hline Main and secondary outcomes & \\
\hline Feasibility ( $n=96)$ & $\mathrm{n}=96$ \\
\hline VAS median score (IQR) & $63(44-78)$ \\
\hline $\mathrm{FS}>50(\mathrm{n}[\%])$ & $62(65)$ \\
\hline Pain felt during examination $(n=96)$ & $\mathrm{n}=96$ \\
\hline VAS median score (IQR) & $31(5-57)$ \\
\hline
\end{tabular}

low, with a median score of 31 (IQR 5-57). Limiting factors to the ultrasound technique were found in 33 patients (34\%). These factors were mainly the following: pain during examination (13 [15\%] patients), technical difficulty (10 [10\%] patients), and obesity (7 [7\%] patients). Other identified factors were perception of technical inexperience for rib ultrasound (4 [4\%] patients), posterior localization of the suspected fracture (2 [2\%] patients), and presence of voluminous breasts limiting ultrasound access (2 [2\%] patients. Six patients had two factors identified. Of them, three had technical difficulty and obesity outlined as limiting factors. Correlation coefficients were obtained for each limiting factor to evaluate whether there was any relation with the degree of feasibility reported by EPs (Table 3). Feasibility categories divided patients in two groups: those with a VAS of 50 or less and those with 51 or more. We found a trend for a weak negative relationship between limiting factors and feasibility. Obesity had a moderate negative correlation, ${ }^{17}$ identified as the main factor that could make rib fracture PoCUS technique less feasible $(\mathrm{r}=-0.39)$.

We found 27 (29\%) patients who were diagnosed with rib fractures on the basis of PoCUS examination who were not diagnosed with standard radiography (Table 4). For 11 patients, a rib fracture was diagnosed with radiography but not with PoCUS (Table 5). Nine of these patients were men with a mean age of 63 years old. No limiting factor was identified for five patients. Patients' pain was identified as a limiting factor for 2 of these 11 patients, as was technical difficulty. One patient had three fractures diagnosed with radiography that were not diagnosed with PoCUS. Three others had two fractures on radiography with a negative PoCUS examination in ED. An FS between 0 and 50 was noted for five patients, and the remaining six patients had an FS superior to 50 .

\begin{tabular}{|c|c|c|c|c|}
\hline \multirow{2}{*}{$\begin{array}{l}\text { Limiting } \\
\text { factors }\end{array}$} & \multirow{2}{*}{$\begin{array}{l}\text { Presence }(+) \text { or } \\
\text { absence }(-) \text { of } \\
\text { limiting factor }\end{array}$} & \multicolumn{2}{|c|}{$\begin{array}{c}\text { Feasibility visual analogue } \\
\text { scale (VAS) score }\end{array}$} & \multirow{2}{*}{$\begin{array}{l}\text { Correlation } \\
\text { coefficient } \\
\text { (r) ( } p \text {-value) }\end{array}$} \\
\hline & & $0-50$ & $51-100$ & \\
\hline \multirow[t]{2}{*}{ Obesity } & + & 7 & 0 & $-0.39(p=0.0001)$ \\
\hline & - & 26 & 62 & \\
\hline \multirow[t]{2}{*}{ Patients' pain } & + & 7 & 6 & $-0.16(p=0.1)$ \\
\hline & - & 26 & 56 & \\
\hline \multirow[t]{2}{*}{ Technical difficulty } & + & 6 & 3 & $-0.22(p=0.03)$ \\
\hline & - & 27 & 59 & \\
\hline \multirow[t]{2}{*}{ Others } & + & 6 & 3 & $-0.22(p=0.03)$ \\
\hline & - & 27 & 59 & \\
\hline
\end{tabular}




\begin{tabular}{|c|c|c|c|}
\hline \multicolumn{4}{|c|}{ Rib view $X$-ray interpreted by radiologist } \\
\hline PoCUS results & $\begin{array}{c}\text { Rib } \\
\text { fracture }\end{array}$ & $\begin{array}{l}\text { Absence of } \\
\text { rib fracture }\end{array}$ & Total \\
\hline Rib fracture & 19 & 27 & 46 \\
\hline Absence of rib fracture & 11 & 36 & 47 \\
\hline Uncertain & 0 & 1 & 1 \\
\hline Total & 30 & 64 & 94 \\
\hline
\end{tabular}

\begin{tabular}{|c|c|}
\hline \multicolumn{2}{|c|}{$\begin{array}{l}\text { Characteristics of patients with negative ultrasound } \\
\text { and positive X-ray }\end{array}$} \\
\hline Men (n [\%]) & 9 (81.8) \\
\hline Age (years) (median and IQR) & $65(54-70)$ \\
\hline \multicolumn{2}{|l|}{ Trauma mechanism (n [\%]) } \\
\hline Fall from height & $8(72.7)$ \\
\hline Sport accident & $1(9.1)$ \\
\hline Motor vehicle accident & $1(9.1)$ \\
\hline Direct blow & $1(9.1)$ \\
\hline \multicolumn{2}{|l|}{ Rib fractures (n [\%]) } \\
\hline 1 & 7 (63.6) \\
\hline$>1$ & $4(36.4)$ \\
\hline \multicolumn{2}{|l|}{ Vital signs (median and IQR) } \\
\hline Heart rate (rate/min) & $75(65-102)$ \\
\hline Respiratory rate (rate/min) & $16(16-20)$ \\
\hline Saturation (\%) & $96(96-98)$ \\
\hline
\end{tabular}

\section{DISCUSSION}

To our knowledge, no previous studies have investigated PoCUS feasibility when performed by EPs to diagnose rib fractures in patients with mTI. Considering a mean FS of 65 on our VAS, our study suggests that the use of PoCUS as technique to diagnose rib fractures in the ED is feasible. EPs reported that patients' pain during PoCUS evaluation was the most significant limiting factor. However, this limiting factor was found in only 13 (14\%) patients on the total number of recruited patients, potentially explaining the global positive evaluation of the ultrasound technique reported by patients. Indeed, the vast majority of patients reported a low score of pain during PoCUS examination. Our results suggest that PoCUS is an acceptable technique that does not seem to be unacceptably painful when rib fractures are suspected. It should be noted that the level of pain experienced during plain radiography evaluation is not zero, considering the mobilization required to obtain adequate views.

Technical difficulty, obesity, posterior location, and voluminous breasts in women were also identified as limiting factors. Obesity had a negative correlation with feasibility but was still moderate. Practice and experience with the specific PoCUS technique may compensate for technical difficulty, but obesity, posterior location, and voluminous breasts in women are identified as nonmodifiable factors that could limit ultrasound evaluation for rib fractures. We suppose that the occurrence of technical difficulty might decrease as the experience of the EP grows with rib fracture PoCUS. Therefore, because our participating EPs were inexperienced with rib PoCUS at the beginning of the study, we believe that the importance of this limiting factor may have been overestimated, and, consequently, the FS results may have been underestimated.

Interestingly, we found that 27 (29\%) patients had rib fractures diagnosed with PoCUS who were not diagnosed with radiography. Given the absence of gold standard in our study, it remains unclear whether these patients represent true or false positives. Nevertheless, the actual evidence on ultrasound sensitivity over radiography concerning rib fracture diagnosis suggests that they were likely to be true positives. ${ }^{11,14,15}$ On the other hand, rib fractures were also diagnosed on X-ray with negative PoCUS results for 11 patients, including multiple fractures in 4 patients. We noted that patients' pain and technical difficulty were identified as the two most important limiting factors. The lack of experience of EPs for rib detection with PoCUS might explain these false-negative results. As the expertise of EPs with rib PoCUS improves, we expect the sensitivity to increase accordingly. Future studies are required to assess this issue.

Some limitations have to be considered when interpreting these results. First, we chose a convenience sample for our study. Underestimation or overestimation of particular groups within the population may have influenced our study results. For example, obese patients could have been underrepresented, leading to easier technique for most patients and fewer identified limiting factors. Second, there is no gold standard for routine rib fracture diagnosis by EP. Even though a CT might be considered in the case of diagnostic tools for rib fractures, we have to take into account the amount of radiation, cost, and accessibility. 
Our study does not have a gold standard as reference, which limits the interpretation of our results on the number of rib fractures diagnosed with PoCUS. Finally, we know that ultrasound is operatordependant. EPs who performed the PoCUS examinations in our study had a mean experience of 9 years using bedside ultrasound as part of their daily practice. They are working in a tertiary trauma centre, where the opportunities to use ultrasound in blunt chest trauma patients are frequent. However, even if they had prior ultrasound exposure, none of the recruiting EPs had previous experience with rib PoCUS. Therefore, we believe that our results could be easily applicable to any clinicians using ultrasound without specific technical experience in rib sonography, making the results of our study widely applicable in the emergency medicine community.

PoCUS indications are growing in number. Within the last few years, PoCUS has become an essential diagnostic modality in our daily practice, and EPs have had numerous opportunities to improve their ability to use it more efficiently. The feasibility of the use of PoCUS for the diagnosis of rib fractures observed in our study might thus be slightly underestimated, as EPs become more experienced and skilled with PoCUS. ${ }^{18}$

\section{CONCLUSIONS}

PoCUS examination to diagnose rib fractures seems to be a feasible and reasonably comfortable technique for ED patients. Rib PoCUS has the potential to augment conventional radiography to diagnose rib fractures. Considering the number of patients diagnosed with rib fractures in the ED, further studies evaluating the potential economic and timesaving impact of using PoCUS for rib fracture diagnosis would be beneficial.

Acknowledgements: The authors want to thank all emergency physicians of the CHU de Québec-Université Laval Enfant-Jésus Hospital for patients' recruitment and Dr. Claude Topping for teaching the training session. As well, they want to thank M. Pier-Alexandre Tardif for his valuable support in reviewing this article.

The authors assert that all procedures contributing to this work comply with the ethical standards of the relevant national and institutional committees on human experimentation and with the Helsinki Declaration of 1975, as revised in 2008. This research received no specific grant from any funding agency, commercial or not-for-profit sectors.
Competing interests: None declared.

\section{SUPPLEMENTARY MATERIALS}

For supplementary material/s referred to in this article, please visit http://dx.doi.org/10.1017/cem.2016.383

\section{REFERENCES}

1. Liman ST, Kuzucu A, Tastepe AI, et al. Chest injury due to blunt trauma. Eur 7 Cardiothorac Surg 2003;23:374-8.

2. Sirmali M, Turut H, Topcu S, et al. A comprehensive analysis of traumatic rib fractures: morbidity, mortality and management. Eur $f$ Cardiothorac Surg 2003;24: 133-8.

3. Kerr-Valentic MA, Arthur M, Mullins RJ, et al. Rib fracture pain and disability: can we do better? $\mathcal{F}$ Trauma 2003;54: 1058-63; discussion 63-4, doi:10.1097/01.TA.0000060262. 76267.EF.

4. Gordy S, Fabricant L, Ham B, et al. The contribution of rib fractures to chronic pain and disability. Am 7 Surg 2014;207:659-62; discussion 62-3, doi:10.1016/j.amjsurg. 2013.12.012.

5. Misthos P, Kakaris S, Sepsas E, et al. A prospective analysis of occult pneumothorax, delayed pneumothorax and delayed hemothorax after minor blunt thoracic trauma. Eur 7 Cardiothorac Surg 2004;25:859-64, doi:10.1016/j.ejcts.2004. 01.044 .

6. Bulger EM, Arneson MA, Mock CN, et al. Rib fractures in the elderly. 7 Trauma 2000;48:1040-6; discussion 6-7.

7. Flagel BT, Luchette FA, Reed RL, et al. Half-a-dozen ribs: the breakpoint for mortality. Surgery 2005;138:717-23; discussion 23-5, doi:10.1016/j.surg.2005.07.022.

8. Battle CE, Hutchings H, Evans PA. Risk factors that predict mortality in patients with blunt chest wall trauma: a systematic review and meta-analysis. Injury 2012;43:8-17, doi:10.1016/j.injury.2011.01.004.

9. DeLuca SA, Rhea JT, O'Malley TO. Radiographic evaluation of rib fractures. A7R Am 7 Roentgenol 1982;138:91-2, doi:10.2214/ajr.138.1.91.

10. Dubinsky I, Low A. Non-life-threatening blunt chest trauma: appropriate investigation and treatment. $A m \mathcal{F}$ Emerg Med 1997;15:240-3.

11. Rainer TH, Griffith JF, Lam E, et al. Comparison of thoracic ultrasound, clinical acumen, and radiography in patients with minor chest injury. $\mathcal{F}$ Trauma 2004;56: 1211-3.

12. Chardoli M, Hasan-Ghaliaee T, Akbari H, et al. Accuracy of chest radiography versus chest computed tomography in hemodynamically stable patients with blunt chest trauma. Chin 7 Traumatol 2013;16:351-4.

13. Cho SH, Sung YM, Kim MS. Missed rib fractures on evaluation of initial chest CT for trauma patients: pattern analysis and diagnostic value of coronal multiplanar reconstruction images with multidetector row CT. $\mathrm{Br} \mathcal{F}$ Radiol 2012;85:e845-50, doi:10.1259/bjr/28575455. 
14. Griffith JF, Rainer TH, Ching AS, et al. Sonography compared with radiography in revealing acute rib fracture. AfR Am 7 Roentgenol 1999;173:1603-9, doi:10.2214/ ajr.173.6.10584808.

15. Kara M, Dikmen E, Erdal HH, et al. Disclosure of unnoticed rib fractures with the use of ultrasonography in minor blunt chest trauma. Eur $\mathcal{f}$ Cardiothorac Surg 2003;24:608-13.
16. Bowen DJ, Kreuter M, Spring B, et al. How we design feasibility studies. Am 7 Prev Med 2009;36:452-7, doi:10.1016/j.amepre.2009.02.002.

17. Cohen J. Statistical power analysis for the behavioral sciences. Hillsdale, NJ: L. Erlbaum Associates; 1988.

18. Heller M, Melanson SW. Applications for ultrasonography in the emergency department. Emerg Med Clin North Am 1997; 15:735-44. 